VDI

KUNSTSTOFFTECHNIK

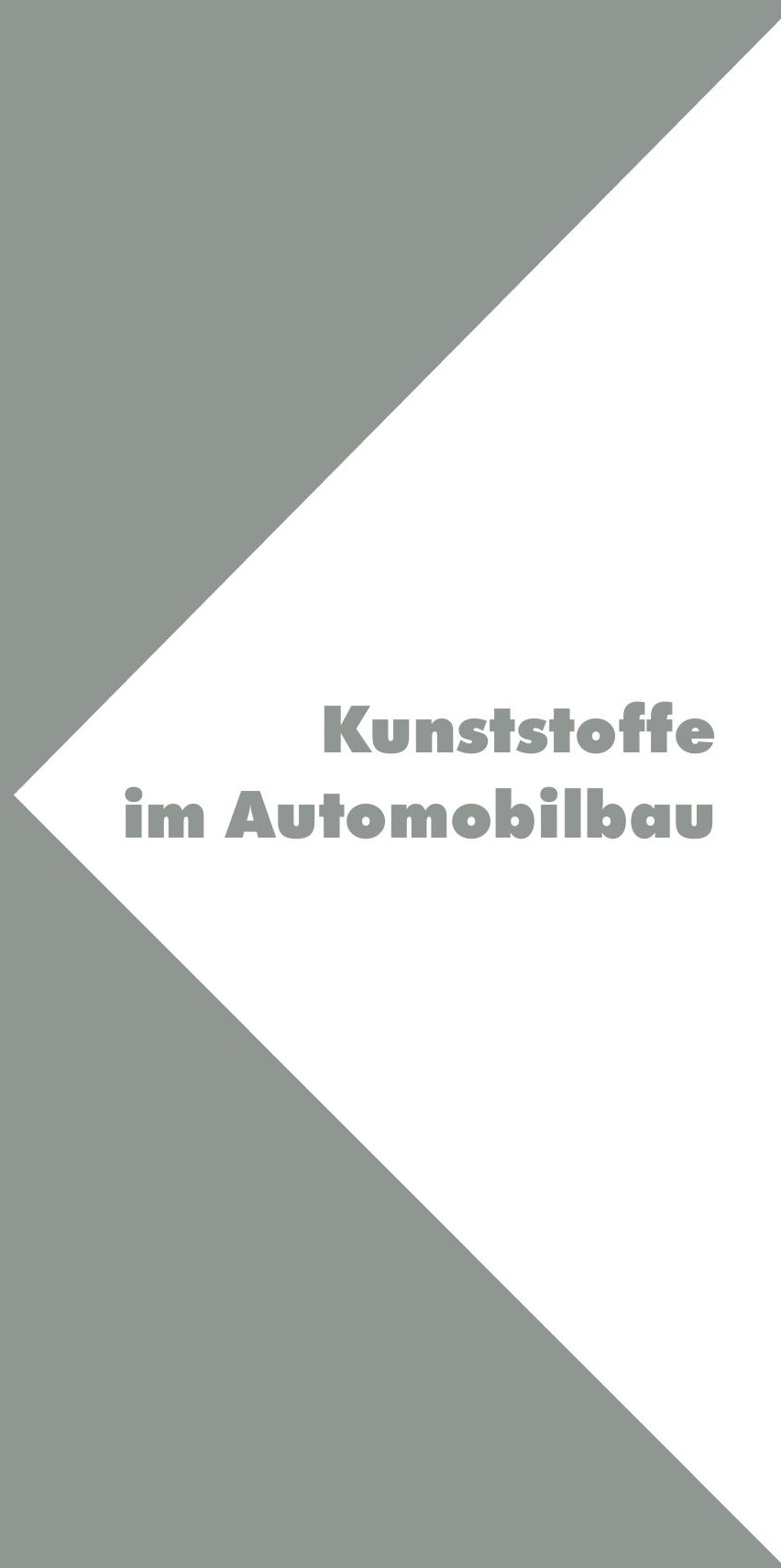


KUNSTSTOFFTECHNIK

\section{Kunststoffe im Automobilbau}

Herausgeber: VDI Wissensforum GmbH

VDI-Gesellschaft Materials Engineering 
Bibliographische Information der Deutschen Bibliothek

Die Deutsche Bibliothek verzeichnet diese Publikation in der Deutschen Nationalbibliographie; detaillierte bibliographische Daten sind im Internet unter http://dnb. ddb. de abrufbar.

\section{Bibliographic information published by the Deutsche Bibliothek}

(German National Library)

The Deutsche Bibliothek lists this publication in the Deutsche Nationalbibliographie

(German National Bibliography); detailed bibliographic data is available via Internet at http:// dnb.ddb.de.

(C) VDI Verlag GmbH · Düsseldorf 2017

Alle Rechte, auch das des auszugweisen Nachdruckes, der auszugweisen oder vollständigen photomechanischen Wiedergabe (Photokopie, Mikrokopie) und das der Übersetzung, vorbehalten.

Printed in Germany

ISBN 978-3-18-234347-9 


\section{Vorwort}

Technische Kunststoffe, faserverstärkte Verbundwerkstoffe sowie multifunktionale Kunststoff-Verbunde unterstützen heute nachhaltig den modernen Automobilbau.

Multifunktionswerkzeuge und automatisierte Verarbeitungsprozesse ermöglichen in vielen Fällen besonders wirtschaftliche Systemlösungen. Die additive Fertigung in Verbindung mit Kunststoffen hat bereits heute ein großes Potenzial, vor allem bei Kleinserien individuelle, maßgeschneiderte Bauteilkonzepte realisieren zu können.

Bei Fahrzeug-Neuentwicklungen stehen heute der Leichtbau, die designtechnische Anmutung des Interieurs, sowie die aktive und passive Sicherheit besonders im Vordergrund.

Innovationen in der Kunststofftechnik beeinflussen direkt die fahrzeugtechnischen Konzepte von morgen. Mischbau mit Kunststoff-Verbunden, Naturfaser-Anwendungen, übergossene und folienhinterspritzte Formteile, LED- und OLED-basierte Lichttechniken, sowie optisch und haptisch optimierte Anzeige- und Bedienkonzepte ermöglichen maßgeschneiderte Systemlösungen im Pkw- und Nutzfahrzeugbau und sichern so langfristig die internationale Wettbewerbsfähigkeit in der Kunststoff- und Automobilbranche ab.

Der Verein Deutscher Ingenieure lädt für den 29. und 30. März 2017 nach Mannheim ein zum internationalen kunststofftechnischen Jahreskongress „Kunststoffe im Automobilbau 2017“. Übersichtsvorträge zu Ressourceneffizienz sowie aus Markt und Forschung, technische Berichte über Kunststoffinnovationen aus dem Pkw- und Nutzfahrzeugbereich, sowie Praxisberichte aus der Kunststoffverarbeitung informieren ausführlich über den heutigen technischen Stand zur Kunststofftechnik im Automobilbau. Eine Fachausstellung von Kunststoff- und Maschinenherstellern sowie ein angegliederter Automobilsalon mit aktuellen Pkws und Nutzfahrzeugen ermöglichen den Fachaustausch am Objekt.

Seien Sie uns herzlich willkommen in Mannheim!

Prof. Dr. Rudolf C. Stauber 
https://doi.org/10.51202/9783182443476-1

Generiert durch IP '172.22.53.54', am 26.04.2023, 03:16:15

Das Erstellen und Weitergeben von Kopien dieses PDFs ist nicht zulässig. 


\section{Inhalt}

\section{Plenum}

Zukunft 2050 - Technologietrends in der Ära der Nachhaltigkeit und der smarten Maschinen

Dr. U. Eberl, SciPress Redaktionsbüro für Wirtschaft, Wissenschaft, Technik, Höhenkirchen bei München

\section{Interieur}

Instrumententafel Nähkleid-Kaschierung - Vom diskontinuierlichen zum kontinuierlichen Prozess

R. Kurz, S. Hobelsberger, H. Auer, BMW AG, Landshut

Methodische Weiterentwicklung der Gewichtsreduzierung von Verkleidungsteilen im Fahrzeuginnenraum - Gegenwärtige und zukünftige Möglichkeiten aus Sicht eines Fahrzeugherstellers

M. Steinbach, J. Maier, Adam Opel AG, Rüsselsheim

New vinyl ink and robotized digital printing process for the fine decoration of an instrument panel made by PVC slush molding

Dr. N. Amouroux, M. El Fouzari, IVY Group, Reims, France

Neue Oberflächen und Anforderungen an die Anwendung von bestehenden Testmethoden

Dipl.-Ing. J. Guenther, Dipl.-Ing. D. Malecha, J. Reinicke, B.Eng.,

Kunststoff-Institut Lüdenscheid, Lüdenscheid

\section{Exterieur}

Der Unterboden - Ein unterschätzter Beitrag zur $\mathrm{CO}_{\mathbf{2}}$-Reduzierung

O. Mende, Volkswagen AG, Wolfsburg

Stoßfänger in Dünnwandtechnologie - ein Update zu Werkstoffen, Verfahren und Technologie-Innovationen

Dipl.-Ing. J. Götzelmann, Magna Exteriors, Sailauf;

Dipl.-Ing. P. Diehl, Magna Exteriors, Esslingen

Entwicklung eines Tankdeckel-Scharnierarms mit Recycling-Material M. Thurmeier, M.Eng., C. Horbas, Dipl.-Ing. (FH) F. Wagner, AUDI AG, Ingolstadt 
Leichte Masse mit Klasse - Heckschürze aus Polyurethan mit äußerst geringer Dichte

Dipl.-Ing. (FH) C. Bauernfeind, Dr. Ing. h. c. F. Porsche AG, Weissach;

Dipl.-Ing. E. Blees, Polytec Group (Polytec Car Styling), Hörsching, Österreich

Active aerodynamic advancements in vehicle underbodies

A. Povinelli, M. Matthews, Magna Exteriors, Troy, Michigan, USA:

Dr. J. J. Laux, Magna Management, Cham, Switzerland;

J. Goetzelmann, Magna Exteriors, Sailauf

Improved crash simulation of endless-fiber-reinforced thermoplastics organic sheets

Dipl.-Ing. M. Franzen, Ford Werke GmbH, Research \& Innovation Center Aachen, Aachen; Dipl.-Ing. G. Oberhofer, MATFEM Partnerschaft Dr. Gese \& Oberhofer, München; Dipl.-Ing. R. Schwarzer, Kirchhoff Automotive Deutschland GmbH, Attendorn

\section{Verfahren}

Innovativer Verarbeitungsprozess thermoplastischer Composites für Porsche Panamera Bremspedal - Endlosfasertechnologie für Sicherheitsbauteile im Fahrzeug

Dipl.-Ing. D. Häffelin, BOGE Rubber \& Plastics, Damme

Energieeffiziente Herstellung von Thermoplast-CFK Bauteilen im einstufigen Direktverfahren

Dr.-Ing. J. Reddemann, Dr.-Ing. H. Seifert, AUDI AG, Ingolstadt

3-D Direktablage von Verstärkungsfasern im Faserblasverfahren - Stand der Technik bei der Naturfaserverarbeitung

R. Korn, M.Sc., BMW AG (via AlphaKraft GmbH), München;

Dr.-Ing. T. Reußmann, TITK e.V., Rudolstadt

Grüner wird’s nicht! Nachhaltig, ökonomisch, sicher: Technische Rezyklate für die Automobilindustrie

Dipl.-Ing. Ansgar Hoffmann, Technische Kunststoffe, Hoffmann + Voss GmbH, Viersen

Tinuvin ${ }^{\circledR} 880$ - novel light stabilizer for automotive interiorapplications

Dipl.-Ing. G. Huber, BASF, Basel, Switzerland

Characterization of microcellular plastics for weight reduction in automotive interior parts

Dr. J. Gómez-Monterde, SEAT SA, Martorell, Spain;

Dipl.-Ing. J. Hain, Volkswagen AG, Wolfsburg;

Prof. Dr. M. LI. Maspoch, Centre Català del Plàstic / Universitat

Politècnica de Catalunya-BarcelonaTech, Terrassa, Barcelona, Spain 


\section{Simulation}

A demonstrator for the experimental assessment of the through-process modeling of injection-molded parts made of short-fiber-reinforced polymers E. Spini, RadiciGroup Performance Plastics, Chignolo d'Isola (Bergamo), Italy; A. Bernasconi, Politecnico di Milano, Milan, Italy

Surface quality: improving the quality perception of molded parts PhD candidate P. Gamonal-Repiso, Dr. J.M. del-Mazo, SEAT S.A, Martorell, Spain; Prof. Dr. M. Sánchez-Soto, Universitat Politècnica de Catalunya-BarcelonaTech, Terrassa/Barcelona, Spain

\section{Technologie}

Leichtbau bei Volkswagen - Über die duroplastische Pultrusion zur großserienfähigen Herstellung faserverstärkter Kunststoffe für einen Lastpfad in der Türstruktur

Dr.-Ing. P. Hörmann, Dipl.-Ing. (FH) K. Bornemann, Dr.-Ing. F. Flueggen,

Dipl.-Ing. H. Herten, Dr.-Ing. V. Hohm, Dr.-Ing. T. Ströhlein,

Volkswagen AG, Wolfsburg

Hohlprofile, Organobleche und LFT-Knoten - Hybridbauteile aus Faserkunststoffverbunden für die automobile Serienproduktion Dipl.-Ing. A. Liebsch, Dr.-Ing. R. Kupfer, Prof. Dr.-Ing. habil. M. Gude, Institut für Leichtbau und Kunststofftechnik, Technische Universität Dresden; P. Müller, M.Sc., Dr.-Ing. N. Andricevic, Dr. Ing. h.c. F. Porsche AG, Weissach

Use Case FVK im Materials Data Space, Digitalisierung der Materialkompetenz als Komplementär zur Industrie $\mathbf{4 . 0}$

Dr.-Ing. R. Schlimper, Dr.-Ing. M. Zscheyge, Prof. Dr.-Ing. P. Michel, Fraunhofer-Institut für Mikrostruktur von Werkstoffen und Systemen IMWS, Halle (Saale)

Two-component air-guide panel manufactured by co-molding and foaming using core-back technology

Dr.-Ing. A. Roch, A. Menrath, Fraunhofer Institute for Chemical Technology ICT, Pfinztal; B. Schmid, BBP Kunststoffwerk Marbach Baier GmbH,

Marbach am Neckar 


\section{Werkstoffe \& Verfahren}

High-Performance Polypropylene - hat PA 6 noch eine Zukunft?

H. Häberle, MAN Truck \& Bus AG, München

Elektrochemische Korrosion und Ihre Vermeidung mit Polyamiden

Dipl.-Ing. G. Prautzsch, Dipl.-Ing. T. Stier,

Dipl.-Ing. T. Coeln, AKRO PLASTIC GmbH Niederzissen

\section{Plenum}

Einsatz von Organoblechen im Fahrzeugunterflur - Kosten- und gewichtsoptimiertes Geländerobustheitspaket

Dipl.-Ing. (FH) R. Apfelbeck, S. Müller, B.Eng. (BA), AUDI AG, Neckarsulm

Innenraumkonzepte - Zukunftsrelevante Designentwicklungen von Fahrzeug-Interiors

J. Friedrich, Car Men $\mathrm{GmbH}$, Idstein

\section{VDI-Fachkonferenz}

\section{Kunststoffe in Nutzfahrzeugen}

\section{Leichtbau}

Lightweight design for increased payload: new ways using polymer composites and physical foaming

L. Jerpdal, M.Sc. M.E., Dipl.-Ing J. Hain, Dr.-Ing. Dipl.-phys. O. Täger, Volkswagen Konzernforschung, Wolfsburg

Carbon-Leichtbau: Leichter und günstiger als Stahl - Gesamtheitliche Betrachtung von Leichtbaupotential \& Prozesskosten von CFK

Dipl. Wiss.-Ing. G. Kalkoffen, CarbonTT, Stade

Entwicklung eines CFK-TP E-Getriebegehäuses

Dipl.-Ing. (FH) M. Kreutzmann, Dr. T. Schneider,

Dipl.-Ing. R. Rademacher, $P+Z$ Engineering $\mathrm{GmbH}$, München 


\section{Kostensenkung}

The use of an alternative material for engine encapsulation for Trucks

T. van den Einden, DAF Trucks, Eindhoven, The Netherlands;

Dipl.-Ing. K. Menke, Johann Borgers GmbH \& Co., Bocholt

A new analytical calculation method for the injectionmolding process of a composite luggage rack holder

M. Bakkal, Istanbul Technical University, Istanbul, Turkey;

O. Otuz, M.Sc., S. Doğru, M.Sc., Istanbul, Turkey

True confidence in thermoplastic composite simulations for any automotive component

W. Schijve, G. Francato, R.Yaldiz, SABIC, Geleen, The Netherlands

Kosteneffizienz durch Einsatz von UV-stabilen Kunststoffen in dynamisch und statisch höchstbelasteten Bauteilen

Dipl.-Ing. (FH) C. Bauer, Dipl.-Ing. (FH) H. Häberle, MAN Truck \& Bus AG, München

Leichtbauträgersystem für den Luftfilter des Mercedes-Benz Actros

Dipl.-Wirt.-Ing. (FH) H. Hauke, BBP Kunststoffwerk Marbach Baier GmbH, Marbach am Neckar; Dipl.-Ing., Dipl.-Wirt.-Ing. J. Horstmann,

LanxessDeutschland $\mathrm{GmbH}$

\section{Zukünftige Kunststoffanwendungen}

Innovative Kunststoffanwendungen für ein kleines Stadtbuskonzept

G. Kopp, O. Deißer, DLR Institut für Fahrzeugkonzepte, Stuttgart;

A. Müller, S. Beyer, Hochschule Esslingen

Innovativer Leichtbau für leichte Nutzfahrzeuge am Beispiel der GFK-Blattzugfeder

Dr.-Ing. J. Stimpfl, Dr.-Ing. J. Asbeck, Mubea Fahrwerksfedern GmbH, Attendorn

\section{Verfahrenstechnik}

Industriegetriebene Initiative zur Standardisierung endlosfaserverstärkter Thermoplaste für den Einsatz in der Automobilindustrie Dr.-Ing. S. Schmeer, Dr.-Ing. D. Scheliga, Institut für Verbundwerkstoffe GmbH, Kaiserslautern 
Goldsponsor:

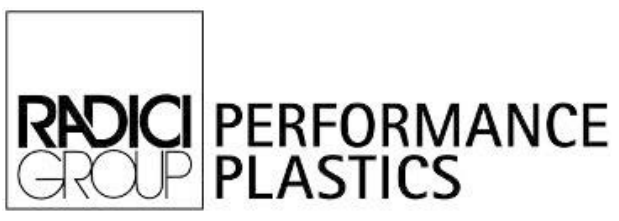

Bronzesponsor:

(S) EVDOIK 